\title{
À la découverte d'un écrivain oublié : Phạm Văn Ký (1910-1992)
}

\section{Giang-Huong Nguyen}

\section{OpenEdition Journals}

\section{Édition électronique}

URL : http://journals.openedition.org/coma/662

DOI : $10.4000 /$ coma.662

ISSN : 2275-1742

\section{Éditeur}

Institut des textes \& manuscrits modernes (ITEM)

\section{Référence électronique}

Giang-Huong Nguyen, « À la découverte d'un écrivain oublié : Phạm Văn Ký (1910-1992) », Continents manuscrits [En ligne], 6 | 2016, mis en ligne le 31 octobre 2016, consulté le 19 avril 2019. URL : http:// journals.openedition.org/coma/662 ; DOI : 10.4000/coma.662

Ce document a été généré automatiquement le 19 avril 2019

\section{(i)

Continents manuscrits - Génétique des textes littéraires - Afrique, Caraîbe, dispora est mis à disposition selon les termes de la licence Creative Commons Attribution - Pas d'Utilisation

Commerciale - Pas de Modification 4.0 International. 


\title{
À la découverte d'un écrivain oublié : Phạm Văn Ký (1910-1992)
}

\author{
Giang-Huong Nguyen
}

1 Phạm Văn Ký (16 janvier $1910-20$ avril 19921) est, parmi les écrivains vietnamiens francophones du xxe siècle, le plus publié en France. Son œuvre témoigne d'un réel talent littéraire, et son époque lui reconnut une place certaine dans le monde des lettres françaises.

2 Avant de devenir romancier et dramaturge en France, il est d'abord connu au Vietnam comme journaliste. À 18 ans, il est déjà rédacteur en chef de deux journaux, l'un à Saïgon, l'autre à Huê, dans lesquels il commence à montrer ses potentialités d'homme de lettres. En 1936, il publie son premier recueil de poèmes en français Une Voix sur la voie. C'est à Paris que sa carrière d'écrivain se développe, abordant tour à tour différents genres littéraires : roman, théâtre et poésie.

3 Produit d'une double culture, vietnamienne et française, Phạm Văn Ký ne cesse de questionner la rencontre des cultures. Une des lignes de force de son œuvre est la confrontation entre le Vietnam et la France, entre les cultures d'Extrême-Orient et celles d'Occident. Son œuvre démontre une excellente maîtrise de la langue française, un vocabulaire riche et précis, une poétique distinctive, inspirée de la pensée extrêmeorientale. Son style - métaphorique, symbolique, abondant en références mythologiques empruntées à différentes cultures - présente aux lecteurs le très vaste arrière-fond de connaissances culturelles de l'écrivain.

\section{Phạm Văn Ký journaliste et poète}

\section{Début de la carrière dans le journalisme}

Issu d'une famille de mandarins, Phạm Văn Ký est le fils aîné d'une famille de douze enfants. Envoyé à Hanoi à partir de 13 ans, il fait ses études en français au lycée du Protectorat, lycée Albert-Sarraut ${ }^{2}$, où il découvre la littérature française. Selon son frère, 
le poète Phạm Hỗ (1926-2007), depuis ses années de lycée, Phạm Văn Ký écrit déjà de la poésie et des romans en vietnamien. Son roman Kiếm hoa, L'Épée et la fleur, est publié en feuilletons dans une revue de l'époque (dont son frère malheureusement a oublié le titre). Un autre, Trên đường thiên lý số 1 , Sur l'autoroute numéro 1, reste inédit ${ }^{3}$. Ces textes sont encore introuvables jusqu'à ce jour.

Pour commencer sa carrière d'écrivain, Phạm Văn Ký se fait connaître par le monde intellectuel au Vietnam d'abord en tant que journaliste. Entre 1932 et 1936, il est rédacteur en chef du journal L'Impartial ${ }^{4}$ qui est un journal populaire à Saïgon à l'époque. Puis à Huê, il collabore avec un journal en vietnamien, le Tràng An báo et devient rédacteur en chef de la Gazette de Huê, une revue littéraire francophone importante de la ville. Enfin, Phạm Văn Ký s'investit exclusivement dans la production littéraire, en langue française, en contribuant, en 1936, à La Nouvelle Revue indochinoise, que Christiane Fournier, romancière, a fondée sur le modèle de La Nouvelle Revue française.

Comparée à celles d'autres écrivains vietnamiens de son époque, cette stratégie n'est pas exceptionnelle. Avant que le genre romanesque ne voie le jour au Vietnam, c'est par la presse que les écrivains vietnamiens du début $d u x^{e}$ siècle se font connaître, s'entraînant à l'écriture en prose et à la production narrative. Faire du journalisme est donc une voie nécessaire pour devenir intellectuel, puis écrivain.

\section{Productions poétiques}

7 Son talent de poète se révèle dès ses vingt ans quand il obtient le premier prix de poésie aux Jeux floraux d'Indochine pour son poème en français « Investiture ». Ce poème sera plus tard publié dans le recueil Une Voix sur la voie en 1936. Phạm Văn Ký a publié au total quatre recueils de poésie ${ }^{5}$ : deux à Saïgon et deux à Paris.

8 Fidèle à la forme poétique française, l'auteur met en avant, dans ce premier recueil, son identité vietnamienne à travers les images, les symboles et les mythes extrême-orientaux. Comme Raphaël Barquisseau le remarque :

Enfin il plonge par des racines profondes dans un monde de rites et de mythes, de traditions et de symboles dont nul Indochinois d'adoption ne pouvait, mieux que cet enfant du sol d'Annam, donner en vers français l'expression fidèle'.

9 L'influence de Mallarmé est très marquée dans la poésie de Phạm Văn Ký. La bande qui recouvre son troisième recueil Fleur de jade $e^{7}$ édité cette fois en France, indique: «Un Mallarmé au pays des Mandarins 8 .» Léon-Gontran Damas confirme cette forte influence mallarméenne :

$\mathrm{Au}$ reste, si ces poèmes font penser à des pastiches, ils sont loin d'être de banales copies. On ne pastiche pas avec une telle réussite sans talent ni sans intelligence, et l'imitation d'un maître avec lequel on se sent quelque affinité est souvent un sûr moyen de se découvrir soi-même. Pas d'art sans exercice. Le pastiche est peut-être la meilleure école du poète. [...] Déjà le premier poème de l'Enceinte Interdite rend un son nouveau et s'efforce à traduire un mystère personnel. Mais c'est surtout dans les poèmes d'amour que les qualités techniques de Phạm Văn Ký, la pureté de sa langue, son sens de la musique du vers et de l'équilibre de la strophe trouvent leur emploi et permettent au poète de traduire une sensibilité à la fois ardente et pleine de fraîcheur. La sincérité du sentiment, l'harmonie, la souplesse du poème préservent Phạm Văn Ký de l'afféterie comme de la banalité9...

En effet, plusieurs vers dans Fleur de jade font écho directement aux poèmes de Mallarmé. Par exemple : 
À soi-même accordé,

Sans qu'Elle n'y consente,

Si furieuse, absente,

La chance encore dé ${ }^{10}$,

renvoie au poème Un Coup de dés jamais n'abolira le hasard de Mallarmé. Ou encore le vers

"Que ma chair se sent triste ${ }^{11}$ » se réfère à « La chair est triste, hélas! et j'ai lu tous les livres $^{12} »$ dans le poème Brise marine. L'idée du vide à travers les images de la neige, du miroir, de la page blanche se présente également dans la poésie de Phạm Văn Ký, comme dans l'extrait suivant :

Le lac opaque attise

Sa si blanche hantise :

Un Cygne d'opéras.

Une feuille est surprise,

Morte, volant au ras

Du Miroir qu'elle brise !

Que de mains se sont tues

D'avoir interrogé

Ce peuple de statues.

... Car ton âme est remplie

De figures d'échecs

Qu'on déplace à coups secs.

Et la courbe accomplie

Des larmes, sur tes joues,

Tarira, si tu joues.

Ce tangage ou roulis

Sur la neige des lits,

Secoue un ventre rose.

Un doigt feuillette en vain

Quelques pages de prose,

Au bord de ce ravin.

Et les sursauts rapides

D'un cœur frivole, impur,

Troublent les eaux limpides.

Restons au pied du mur

D'où le jour lointain tombe,

(Si près de notre tombe !) ${ }^{13}[. .$.

De mentalité taoïste, c'est peut-être par sa conception du vide que Phạm Văn Ký trouve le plus d'affinités avec la poésie mallarméenne. Mallarmé, particulièrement dans «Un Coup de dés... ", crée un espace typographique où le vide et la circulation des signes lui permettent de «Donner un sens plus pur aux mots de la tribu..$^{14}$ ». Affranchis de leur contexte habituel et de leur usage ordinaire, les mots peuvent enfin délivrer leurs ressources cachées et accèdent ainsi à une dimension pleinement poétique, presque mystique, au sein d'un langage entièrement renouvelé. De manière similaire mais à un niveau plus philosophique, le vide dans le taoïsme donne les potentialités pour l'existence du plein, il est la source même du plein.

Dans l'ordre du réel, le Vide a une représentation concrète : la vallée. Celle-ci est creuse, et, dirait-on, vide, pourtant elle fait pousser et nourrit toutes choses; et portant toutes choses en son sein, elle les contient sans jamais se laisser déborder et $\operatorname{tarir}^{15}$.

13 Ainsi se forme dans la poésie mallarméenne une esthétique du métalangage qui va jusqu'à l'hermétisme. Cette esthétique constitue un élément important dans les réflexions de Phạm Văn Ký sur sa création littéraire, qui doit être liée en particulier à son problème de 
bilinguisme franco-vietnamien. Elle dépasse d'ailleurs le domaine de la poésie pour déterminer la vocation esthétique et philosophique dans toute l'écriture romanesque postérieure de Phạm Văn Ký.

Phạm Văn Ký occupe alors une place importante dans le monde littéraire vietnamien avant son départ pour la France, d'abord en tant que journaliste, puis en tant que poète. En particulier, "en décidant d'écrire en français, l'écrivain s'engage dans la voie des intellectuels qui ont compris qu'après 1862, date du début de l'occupation française, le pays doit s'occidentaliser pour pouvoir faire face à l'adversaire avec ses propres armes ${ }^{16}$ . " L'élite vietnamienne place ses espoirs en Phạm Văn Ký comme en d'autres jeunes intellectuels pour mener le Vietnam vers une révolution sociale et culturelle qui, selon elle, serait la condition nécessaire pour l'indépendance du pays. Une correspondance de Nguyễn Giang (1910-1969), peintre et poète vietnamien, révèle ce souhait :

Eh, oui ! mon cher Ký, que faisons nous? nous travaillons, n'est ce pas ? Et pourquoi travaillons nous? Pour gagner notre vie... mais au fond, derrière ce but réel et immédiat, nos [sic.] visons tous encore un autre but : le bien du pays, le relèvement matériel et surtout moral et intellectuel de tout un peuple. C'est encore là une vérité que je conçois non par excès d'optimisme mais par une juste interprétation de nos activités. Eh bien, je pense que dans l'état actuel de notre société, ce qui peut le plus contribuer à ce relèvement, c'est un « Mouvement », quel qu'il soit, pourvu que ce soit un mouvement qui ait un caractère national. Un mouvement littéraire et artistique national, voilà la chose à laquelle j'ai rêvé depuis longtemps, à laquelle je travaille, à laquelle je me permets de vous associer par avance*. Ce n'est pas sans avoir longuement médité, je m'adresse à vous pour que vous [mot illisible] aussi, avec votre "plume alerte", à donner à ce mouvement toute l'ampleur désirable. J'ai beaucoup pensé à vous, à vous et à Nguyen Tien Lang qui êtes les deux plus beaux représentants de cette nouvelle famille d'écrivains annamites qu'on appelle « les écrivains annamites de langue française ${ }^{17}[. .$.$] .$

On ne sait pas ce qu'a répondu Phạm Văn Ký à cette lettre. Mais dans sa nouvelle L'Ogre qui dévore les villes, publiée en 1946 dans Les Temps modernes, l'écrivain s'exprime ainsi :

Aussitôt dépassé l'enceinte de bambous, j'appréhendai mon dépaysement dans une ville étrangère. Mais en compensation, la nature de ma mission me conférait ma dignité toute neuve [...] J'invoquai la silhouette légendaire de Tam-Tang qui, chargé de ramener, de l'Ouest, le manuscrit de Bouddha, avait été transfiguré par ce périple, non sans avoir subi maintes tribulations. D'avance, je me plus à grossir l'importance de mon mandat, à m'élever à la hauteur d'un chercheur de véritée ${ }^{18}$ !

Cette réponse ne va pas à l'encontre de ce que lui avait suggéré son grand ami Nguyễn Giang. L'histoire ne voit pas se réaliser ce «Mouvement » que ce dernier a évoqué dans ses correspondances, mais le souhait d'une évolution culturelle et intellectuelle pour relever le Vietnam de la situation de subordination est réel chez les érudits vietnamiens nationalistes comme Nguyễn Giang et Phạm Văn Ký. Abandonnant sa position au centre du champ littéraire vietnamien, Phạm Văn Ký nourrit alors l'ambition de s'affirmer et d'affirmer l'identité nationale devant le public de la métropole.

\section{Phạm Văn Ký dramaturge et romancier}

17 Arrivé en France en $1939^{19}$, Phạm Văn Ký s'inscrit à l'université de la Sorbonne pour des études en littérature, puis prépare également, à l'Institut des Hautes Études chinoises, une thèse en sciences religieuses. Cependant, il est obligé d'interrompre sa thèse à cause de la Seconde Guerre mondiale et de la mort de son directeur de thèse, Marcel Mauss ${ }^{20}$. 
Pour survivre, il doit être tour à tour professeur de français dans un cours privé à Neuilly et rédacteur à la préfecture de la Seine, tout en écrivant des poèmes et des nouvelles. On reconnaît la même stratégie que celle qu'il développait au Vietnam : se faire connaître par le monde des lettres parisien, en se faisant publier par les journaux, puis par les revues littéraires.

Le plus ancien article conservé dans son fonds d'archives date du 4 octobre 1945. C'est une nouvelle intitulée : «Ba De ou les Parents terribles ${ }^{21}$ ». Le public français pouvait au début être intéressé par l'aspect exotique et la couleur folklorique de ses nouvelles. Mais bien vite, Phạm Văn Ký évoque dans celles-ci des problèmes plus profonds issus de sa double culture: le déchirement entre deux civilisations, la nostalgie de son pays d'origine, et de manière plus universelle, les conflits entre les différentes cultures et la situation difficile de l'être humain qui éprouve ces conflits. Pendant les années 1940 et 1950, il fait connaitre son nom en publiant sans cesse des nouvelles et des articles de critique sur les arts en Extrême-Orient (Vietnam, Chine, Japon) ${ }^{22}$. L'écrivain collabore à différentes revues importantes de l'époque comme Esprit, Les Temps modernes, Les Cahiers du Sud, La Nef, Synthèses, L'Âge nouveau, Les Nouvelles littéraires, Preuves, Paru...

À son tour, la presse lui consacre des articles. À partir de 1946, on entend parler de Phạm Văn Ký parmi d'autres écrivains francophones des colonies dans les articles consacrés à l'Union française, fondée la même année, et dans les journaux de tendance socialiste. L'intérêt de la presse pour ces écrivains est lié au slogan de l'Union française prônant la solidarité et l'égalité entre la métropole et ses colonies. Le journal Le Populaire, organe du Parti socialiste SFIO, fait paraître dans des numéros successifs en 1947, dans la rubrique intitulée "Voix de l'Union française » des interviews de Phạm Văn Ký, Léopold Sédar Senghor et Jean Amrouche, menées par le poète Georges-Emmanuel Clancier. La raison de ces interviews est bien exprimée dans l'article portant sur Phạm Văn Ký :

À l'heure où l'Union française se trouve gravement et de toutes parts menacée, nous avons pensé qu'il serait utile de demander à un Annamite, un Sénégalais, un Berbère, leur opinion sur les rapports de leur pays et de la métropole dans le présent et dans l'aveniri ${ }^{23}$.

\section{Productions théâtrales}

21 C'est en 1944 que Phạm Văn Ký s'introduit dans le domaine du théâtre avec ses trois petites pièces en un acte incluses dans Fragments annamites, programme de théâtre du Centre national des étudiants de la France d'Outre-Mer, daté du 25 juin 1944. Son poèmeballet est dansé par Janine Charrat en 1946. Il publie également plusieurs articles sur le théâtre vietnamien et le théâtre chinois dans Le Magazine du spectacle en 1946, puis 1948. Il entre à la Radiodiffusion française en 1947 avec sa pièce radiophonique La Muraille de Chine.

Les pièces de théâtre de Phạm Văn Ký portent sur la thématique des échanges culturels, en s'appuyant sur des contextes historiques précis. C'est le cas de la série radiophonique Premières Ambassades entre l'Europe et l'Asie ${ }^{24}$. Notamment, la 4 émission : France-Viet-Nam : Alexandre de Rhodes qui relate les premières prises de contact, en 1627, à la cour vietnamienne du jésuite Alexandre de Rhodes, apportant les premières nouveautés venant de l'Europe. Dans le sens inverse et symétrique, la $5^{\mathrm{e}}$ émission : Viet-Nam - France: Phan Thanh Gian à la Cour de Napoléon III raconte le voyage du premier ambassadeur vietnamien Phan Thanh Gian se rendant en France en 1863 pour envisager la rétrocession 
de trois provinces de la Cochinchine. Sans se limiter au contexte vietnamien, cette série s'ouvre aussi à d'autres pays asiatiques qui entraient en contact avec des pays d'Europe : Hollande-Japon, Russie-Japon, Angleterre-Chine, France-Laos, USA-Siam, AngleterreBirmanie, France-Inde, Portugal-Inde. Plusieurs autres œuvres sont inspirées de l'histoire de la Chine telles que Confucius ${ }^{25}$, Tchao-Kiun ${ }^{26}$ ou La Muraille de Chine ${ }^{27}$; certaines autres s'inscrivent dans le contexte nippon comme la pièce Hiroshima et l'île du bonheur ${ }^{28}$. Au moins deux pièces sont consacrées au poète indien Rabindranath Tagore.

Dans ces écrits, la structure de la dualité constitue une métaphore textuelle de la problématique des confrontations culturelles. Cette structure consiste à mettre en opposition deux temps, deux espaces ou deux types de personnages, représentant deux systèmes de valeurs différents. Elle n'est d'ailleurs pas inconnue dans les romans francophones portant sur l'interculturalité. Dans les pièces dramatiques de cet écrivain, la structure de la dualité est encore plus visible. Elle met alors en scène un contexte inévitable où deux cultures se rencontrent et s'affrontent intensément. Là est l'origine de la tension qui nécessite un dénouement final : recherche de la compréhension de l'autre culture ou volonté de conciliation entre différentes valeurs.

La pièce L'Architecte et le Géomancien met par exemple en opposition un architecte et un géomancien. Ceux-ci participent à la construction d'un village chinois en Europe, à Cassel (en 1781). L'un mobilisant un esprit cartésien et l'autre fonctionnant comme un artiste extrême-oriental, ils n'arrivent pas à se comprendre. Le village risque de ne jamais voir le jour à cause des malentendus fondamentaux entre un Occidental et un Extrême-Oriental. La controverse a ses témoins des deux côtés : tour à tour, apparaissent les personnages les plus divers d'Orient et d'Occident, de l'Antiquité ou des temps modernes : de Marco Polo à Walt Whitman, et de l'empereur K'ang Hi à Rabindranath Tagore.

Dans une pièce policière, La Malédiction de sept félicités ${ }^{29}$ le dramaturge met en scène un détective anglais face à une meurtrière asiatique Mme Lin Tsou, une antiquaire chinoise. Celle-ci a assassiné successivement cinq collectionneurs d'une statuette Quantine ${ }^{30}$. Elle a eu recours à une ruse mystérieuse de l'Extrême-Orient qui échappe au détective européen : elle dissimule, sous son ongle démesuré, une aiguille avec laquelle elle injecte un poison mortel à ses victimes. Ironiquement, un cliché exotique sur l'Indochine nourrit ici le genre policier. On reconnait l'obsession des Européens pour les "horrifiques " images de mains des lettrés "annamites» que l'on voit sur les cartes postales d'Indochine. Ces images participent également à entretenir délicieusement le fantasme d'un péril jaune tel que l'incarne l'ignoble docteur Fu Manchu, le terrifiant héros de Sax Rohmer.

\section{Productions romanesques}

Lors de la publication de son premier roman, Phạm Văn Ký n'est alors pas inconnu dans le monde des lettres françaises. Une annonce de son roman Frères de sang le confirme :

Frères de sang, roman que les Éditions du Seuil vont publier dans la collection «Pierres Vives ", a pour auteur un jeune Vietnamien, Phạm Văn Ký, dont on a pu lire des nouvelles dans Les Temps modernes, Esprit, La Revue internationale ${ }^{31}$... Lettres françaises, La Revue des livres, La Revue internationale ${ }^{32}$ ) et est mentionné par le jury du prix des Critiques en 1947. 
28 Tout en travaillant régulièrement comme auteur pour la Radio et en remportant différents prix pour sa production dramatique, Phạm Văn Ký continue à publier des romans: Celui qui régnera (Grasset, 1954), Les Yeux courroucés (Gallimard, 1958), Les Contemporains (Gallimard, 1959). Le sommet de son succès sera le Grand Prix du roman de l'Académie française attribué à son roman Perdre la demeure en $1961^{33}$.

29 Au fur et à mesure de sa création, Phạm Văn Ký se libère de l'espace vietnamien pour évoquer un problème plus universel: la rupture qui se produit lorsqu'un système de valeurs s'affronte à un autre, dans tout espace culturel. Depuis son deuxième roman, Celui qui régnera (1954), même si l'histoire se passe toujours dans le contexte vietnamien, l'écrivain s'exprime ainsi :

- Quel but avez-vous poursuivi en l'écrivant?

- D'abord, construire une histoire cohérente avec un centre d'intérêt, des péripéties et une progression dramatique. Ensuite, sous ce " prétexte ", raconter une rupture, en dépeignant les mœurs et les coutumes d'une période en voie de disparition. Mais en même temps, veiller à ne pas tomber dans ce qu'on appelle, en mauvaise part, la couleur locale, et à ce que ces motifs dits folkloriques forment la trame même du conflit. En définitive, le but que je me suis proposé se réduit à intéresser, à introduire le lecteur européen dans un univers qui le dépayse sans le dérouter, et cela en lui montrant que, malgré des différences de surface, ou même des différences de comportement, l'homme se trouve toujours devant les mêmes problèmes, à quelque pays qu'il appartienne, et où qu'ils se posent ${ }^{34}$.

Cette problématique de rupture continue à être explorée dans les romans suivants de Phạm Văn Ký mais dans d'autres espaces culturels : de la Chine (Les Yeux courroucés, Les Contemporains) et du Japon (Perdre la demeure).

Enfin, c'est dans l'espace énonciatif de Paris que Phạm Văn Ký s'interroge sur une conciliation absolue des conflits idéologiques et culturels entre l'Extrême-Orient et l'Occident. Cette question s'est posée en permanence dans ses manuscrits inédits Paris, qu'as-tu fait de nous? et La Robe de brocart. C'est alors dans son roman Des Femmes assises çà et là (publié en 1964) que Phạm Văn Ký propose une nouvelle manière de réfléchir sur l'interculturalité et la diversité culturelle, en se fondant sur la philosophie du dualisme du Yi King ou Le Livre des Mutations ${ }^{35}$. Selon lui, l'Extrême-Orient et l'Occident existent et évoluent dans une "signification d'ensemble ${ }^{36}$ " qui implique que toutes les choses apparemment disparates, les unes visibles, les autres invisibles, sont interdépendantes dans la logique de l'harmonie et de l'alternance entre le yin et le yang. L'Extrême-Orient et l'Occident sont à l'intérieur du cercle du Faîte suprême, comme la partie blanche représentant le yin et la partie noire le yang. Ils «s'opposent, alternent, se succèdent à l'ouvrage et se parfont mutuellement, harmonieusement ${ }^{37}$ " pour former une sorte de symbiose de cultures.

\section{L'oubli}

32 À partir de 1970, on n'entend plus parler de Phạm Văn Ký : il ne publie plus, seule sa pièce de théâtre Le Rideau de pluie est montée et jouée en 1974.

Un événement important de sa vie personnelle et de sa carrière d'écrivain, sans aucun doute, est son unique retour au Vietnam en 1970. Phạm Văn Ký fait partie d'une délégation des Việt Kiều - Vietnamiens résidant à l'étranger - et retourne au Vietnam invité par le Front de la Patrie à la commémoration de la révolution d'Août (le 19 août 1945) et de la fondation de la République démocratique du Vietnam (le 2 
septembre 1945). De retour de ce voyage à Paris, il écrit son essai Le Défi vietnamien ${ }^{38}$, racontant son séjour au Vietnam, les rencontres qu'il a faites. L'essai révèle certes beaucoup d'émotions après ses trente ans d'une vie d'expatrié : trente ans avec beaucoup de changements radicaux, du régime politique à la réalité sociale et culturelle. Dans cet essai, l'écrivain prend position comme observateur, mais bien concerné par la réalité de la société de l'État socialiste du Nord du Vietnam; et non comme un observateur embarrassé et n'agissant pas, à l'image du narrateur de son premier roman Frères de sang en 1947.

Son frère Phạm Hỗ, dans un texte sur l'écrivain, a affirmé le lien entre ce retour au Vietnam et le retrait de Phạm Văn Ký du monde éditorial :

Pendant ses dernières années, il a une vie difficile. Car de son retour au pays jusqu'à son décès, les grands éditeurs français ne publient aucun de ses manuscrits. Même pour un projet de roman qu'il a signé avec Gallimard avant son voyage au Vietnam, à son retour, il apprend que l'éditeur cherche un prétexte pour l'annuler ${ }^{39} .$.

En réalité, ceci est dû à un changement qui se manifeste dans l'écriture de Phạm Văn Ký depuis cette date. À partir de 1970, ses manuscrits semblent porter notamment sur l'histoire du Vietnam. Ceux-ci ne sont pas datés mais les événements évoqués dans les intrigues peuvent nous permettre de les localiser dans le temps. Par exemple, le dernier volume du roman L'Ennemi qui nous ressemble $e^{40}$ parle des boat people, un événement historique qui ne se produit qu'à partir de 1975. Phạm Văn Ký serait-il rejeté par les éditeurs parisiens, son retour au Vietnam en 1970 portant une signification politique ? Ou simplement, préfèrent-ils que l'écrivain reste dans sa ligne de force, d'écrire sur les problèmes d'acculturation et de rencontres culturelles? Alors, lui-même qui avoue être "entêtét1 ", accepterait-il d'infléchir sa vocation d'écriture et d'obéir à l'attente des éditeurs?

Un fragment d'une lettre écrite par Phạm Văn Ký révèle les explications de l'écrivain luimême sur le fait qu'il ne publie plus :

Que l'on garde sa foi dans des manuscrits qui n'ont pas encore essuyé les feux des comités de lecture, ou qui n'ont pas été retenus en leur temps, pour des raisons tactiques - cf.: les prix littéraires, - ou idéologique - «Ne politique pas avec le Mongol », - on leur attribue une pérennité qui ne leur appartient peut-être pas, mais dont on a besoin. Or, le "repêchage " in extremis de textes refusés à une certaine époque, nécessiterait, entre les parties intéressées, des mutations concomitantes d'une extraordinaire subtilité et des coïncidences qui relèveraient du miracle. Et, au bout du compte, un jeu que l'on n'abat jamais risque de perdre toute valeur.

Derrière moi, s'étire un désert de treize ans, qui ressemble à un nombre à longue queue, à partie décimale illimitée, sinon au « mauvais infini » de Hegel : l'infini dont on ne sort pas. Au vrai, l'auteur n'est innocent de rien. Je ne traverse pas ce désertlà : je le fais. Il y a que j'ai fort peu d'entrailles pour la course aux éditeurs et aux directeurs de théâtre. Il suffisait à ma peine d'avoir écrit à deux de ceux-ci et à trois de ceux-là, tandis que plus entêtés que moi, du moins en ce domaine, en accomplissent allégrement les deux tours complets, tels les bolides dans l'espace courbe d'Einstein, avant de [fin de la page $]^{42}$.

37 En raison de points de vue politiques non partagés, ajoutés à son tempérament opiniâtre, comme il l'avoue, Phạm Văn Ký se lasse de démarcher les éditeurs. C'est aussi peut-être qu'à partir de son voyage au Vietnam, il tente de se tourner vers le champ littéraire de son pays natal. L'auteur réapparait en effet dans les revues littéraires vietnamiennes avec des poèmes qui chantent le président Hồ Chí Minh, la reconstruction du pays socialiste, les combats héroïques du Viêt-Minh. En 1993, un an après sa mort, le Comité national des 
Écrivains vietnamiens regroupe ses poèmes parus au Vietnam depuis 1970 pour publier un recueil sous le titre Đường về nước, Le Chemin vers le pays natal. Pourtant, ce retour ne semble pas si facile. Les intellectuels et les écrivains vietnamiens ne parlent pas de lui, les dictionnaires et les anthologies d'écrivains vietnamiens ne le mentionnent pas. Jusqu'à la fin de sa vie, Phạm Văn Ký reste un "rôdeur de confins ${ }^{43}$ ", tombé aujourd'hui dans l'oubli, en lequel s'enchevêtrent deux cultures, deux champs littéraires de la France et du Vietnam.

\section{BIBLIOGRAPHIE}

Pham, Van Ky, Celui qui régnera, Paris, Bernard Grasset, 1954.

Pham, Van Ky, Des Femmes assises çà et là, Paris, Gallimard, 1964.

Pham, Van Ky, Đường về nước, Hà Nội, Nxb. Hội nhà văn, 1993.

Pham, Van Ky, Fleur de jade, Paris, Éd. du Livre moderne, 1943.

Pham, Van Ky, Frères de sang, Paris, Éd. du Seuil, 1947.

Pham, Van Ky, L'Homme de nulle part, Paris, Fasquelle, 1946.

Pham, Van Ky, Les Contemporains, Paris, Gallimard, 1959.

Pham, Van Ky, Les Yeux courroucés, Paris, Gallimard, 1958.

Pham, Van Ky, Perdre la demeure, Paris, Gallimard, 1961.

Pham, Van Ky, Poème sur soie, Paris, Éd. Euros (impr. Daragnès), 1961.

Pham, Van Ky, Une Voix sur la voie, Saïgon, Aspar, 1936.

Pham, Van Ky, La Malédiction des sept félicités, Institut National de l'Audiovisuel, http:// www.ina.fr/audio/PHD88015101/la-malediction-des-sept-felicites-audio.html, consulté le 9 novembre 2015.

Pham, Van Ky, L'Ogre qui dévore les villes, in Les Temps modernes, $\mathrm{n}^{\circ}$ 14, Paris, 1946, p. 238-252.

Pham, Van Ky, « Mes Historiographes de droite et de gauche », in La Revue théâtrale : revue internationale du Théâtre, $n^{\circ}$ 25, Paris, Bordas, 1953, p. 67-73.

«Ils ont choisi la langue française pour s'exprimer », in Panorama Chrétien, Paris, septembre 1964.

«La boîte aux lettres ", in Opéra : l'hebdomadaire du théâtre, du cinéma, des lettres et des arts (de Jacques Chabannes), Paris, 14 mai 1947.

Aubarede, Gabriel, « Instantané Phạm Văn Ký », Les Nouvelles Littéraires, n 1415, Paris, 15 août 1958, p. 7.

Beaumarchais, Jean-Pierre de, Couty, Daniel, Rey, Alain, Dictionnaire des littératures de langue française. 3, Paris, Bordas, 1994.

Beaumarchais, Jean-Pierre de, Couty, Daniel, Rey, Alain, Dictionnaire des écrivains de langue française, Paris, Larousse, 2001. 
Cheng, François, Vide et plein. Le langage pictural chinois, Éd. du Seuil, Paris, 1979.

Clancier, Georges-Emmanuel, « Vietnam », in Le Populaire : l'organe du Parti socialiste S.F.I.O., Paris, vendredi 25 avril 1947.

Damas, Léon-Gontran, Latitudes françaises. I, Poètes d'expression française : 1900-1945, Paris, Éd. du Seuil, 1947.

Demougin, Jacques, Dictionnaire de la littérature française et francophone, Paris, Larousse, 1987.

Ho, Huy, « Phạm Văn Ký », in DO Duc Hieu (ed.), Từ điển văn học : bộ mới, Hà Nội, Thế giới, 2004, p. 1376.

Joubert, Jean-Louis, Petit Guide des littératures francophones, Paris, Nathan, coll. « Les petites références ", 2006.

Luthi, Jean-Jacques, VIATTE, Auguste, ZANANIRI, Gaston, Dictionnaire général de la francophonie, Paris, Letouzey et Ané, 1986.

Mallarmé, Stéphane, Poésies, Paris, Bookking International, 1993.

Nguyen, Cong Ly, « Parcours littéraire de Phạm Văn Ký à travers la presse francophone » (en vietnamien), Bulletin des Sciences Sociales et Humaines, Université des Sciences Sociales et Humaines, Ho Chi Minh vielle, nº 52, 2011, p. 3-11.

- « Le sujet clivé dans la nouvelle Le Fantôme de la précision de Phạm Văn Ký », in Mondes Francophones, http://mondesfrancophones.com/espaces/psyches/le-sujet-clive-dans-la-nouvellele-fantome-de-la-precision-de-Pham-van-ky/, consulté le 09 novembre 2015.

- L'Institution de la littérature vietnamienne francophone, Paris, Publibook Editions, 2013.

Nguyen, Lucy Hong Nhiem, L'Échiquier et l'antinomie Je/Moi comme signe et substance du conflit Occident/Extrême-Orient dans les œuvres de Phạm Văn Ký, thèse soutenue à University of Massachusetts, Amherst en 1982.

Nguyen, Vy, Văn thi sĩ tiền chiến: hồi ký văn học, (1970), Hà Nội, Nxb. Hội nhà văn, 2007.

Pham, Van Quang, «L'écrivain francophone Phạm Văn Ký : une identité paradoxale », in Mondes Francophones, http://mondesfrancophones.com/debats/francophonies-et-theories/lecrivainfrancophone-Pham-van-ky-une-identite-paradoxale/, consulté le 09 novembre 2015. - L'Ordre des mots dans les romans francophones de Cung Giu Nguyen, Phạm Văn Ký et Pham Duy Khiem : étude linguistique, stylistique et poétique de l'inversion du sujet et de la place des compléments et des adverbes, thèse soutenue à l'Université de Toulouse-Le Mirail, Toulouse en 2007.

Vuong-Riddick, Thuong, « Corps et acculturation selon Phạm Văn Ký », Présence francophone, n ${ }^{\circ}$ 18, printemps 1979, p. 165-176.

Vuong-Riddick, Thuong, « Le Drame de l'occidentalisation dans quelques romans de Phạm Văn Ký », Présence francophone, $n^{\circ} 16$, printemps 1978, p. 141-152.

Yeager, Jack Andrew, The Vietnamese Novel in French: a literary Response to Colonialism, Hanover (NH), University of New Hampshire by University Press of New England, 1987.

- « Writing from Exile: Phạm Văn Ký's Imagined Returns to Viet Nam », in Barbara Tran et Rebekah Linh Collins (eds.), Vietnam: beyond the frame, Ann Arbor (MI), University of Michigan, mis en ligne en 2004, consulté le 21 août 2014. URL : http://hdl.handle.net/2027/

spo.act2080.0043.425. 


\section{NOTES}

1. Selon son acte de décès, transcrit à la mairie de Maisons-Alfort.

2. Le niveau "lycée » ici inclut le petit lycée - équivalant aujourd'hui à la fin de l'école primaire et au collège -et le grand lycée équivalant aujourd'hui au lycée.

3. Huy Ho, «Phạm Văn Ký ", in Duc Hieu Do (éd.), Từ điển văn học : bộ mới, Hà Nội, Thế giới, 2004, p. 1376. Ho Pham, « Những kỷ niệm về anh tôi ", in Van Ky Pham, Đường về nước, Hà Nội, Nxb. Hội nhà văn, 1993, p. 53.

4. Journal de tendance constitutionnaliste. Le Parti constitutionnaliste fondé en 1923 se veut le champion de la modernité et veut faire appel à la «France démocratique ». Expression de la bourgeoisie cochinchinoise, il réclame plus de libertés, plus de place pour les Annamites dans les administrations et la direction du pays, notamment pour ceux qui ont fait leurs études en métropole.

5. Dans la publication Fleur de jade, les recueils Poèmes d'amour (4-COL-25/84), [Annam]: Somme de ferveur $\left(4^{\circ}-\mathrm{COL}-25 / 81\right)$ sont annoncés à paraître (chez Denoël pour le premier) mais les publications restent introuvables jusqu'à ce jour. Leurs exemplaires pré-édités se trouvent dans le fonds d'archives. Un poème «Somme de ferveur » a été publié dans La Revue internationale, $\mathrm{n}^{\circ} 7$, août 1946.

6. Préface de Raphaël Barquisseau au livre de Van Ky Pham, Une voix sur la voie, Saïgon, Aspar, 1936, p. 8.

7. Van Ky Pham, Fleur de jade, Paris, Éd. du Livre moderne, 1943.

8. Léon-Gontran Damas, Latitudes françaises. I, Poètes d'expression française : 1900-1945, Paris, Éd. du Seuil, 1947, p. 219.

9. Ibid., p. 219. L.-G. Damas a cité un certain Roger Beillon sur qui nous n'avons trouvé malheureusement aucune information biographique.

10. Van Ky Pham, Fleur de jade, op. cit., p. 13.

11. Ibid., p. 19.

12. Stéphane Mallarmé, Poésies, Paris, Bookking International, 1993, p. 31.

13. Van Ky Pham, « Sauvés de l'ombre... », Fleur de jade, op. cit., p. 91-95.

14. Stéphane Mallarmé, « Le tombeau d'Edgar Poe », Poésies, op. cit., p. 81.

15. François Cheng, Vide et plein. Le langage pictural chinois, Éd. du Seuil, Paris, 1979, p. 29.

16. Thuong Vuong-Riddick, «Le Drame de l'occidentalisation dans quelques romans de Pham Van Ky ", op. cit., p. 142.

17. Lettre de Nguyen Giang à Pham Van Ky datée du 4 juin 1936. Fonds Pham Van Ky, BnF : $4^{\circ}-$ COL-25/177.

18. Van Ky Pham, L'Ogre qui dévore les villes, Les Temps modernes, $\mathrm{n}^{\circ}$ 14, Paris, 1946, p. 241.

19. Van Ky Pham, Paris, qu'as-tu fait de nous?, manuscrit dactylographié, Fonds Phạm Văn Ký, BnF : $4^{\circ}$-COL-25/8, p. 11.

20. Jack Andrew Yearger, The Vietnamese Novel in French: a literary Response to Colonialism, Hanover (NH), University of New Hampshire by University Press of New England, 1987, p. 171.

21. Fonds Pham Van Ky, BnF : $4^{\circ}-\mathrm{COL}-25 / 122$.

22. Fonds Pham Van Ky, BnF : $4^{\circ}$-COL- 25/105.

23. Georges-Emmanuel Clancier, "Vietnam», Le Populaire: l'organe du Parti socialiste S.F.I.O. [Section Française de l'Internationale Ouvrière], Paris, vendredi 25 avril 1947.

24. Fonds Pham Van Ky, BnF : $4^{\circ}$-COL-25/65 (1-10). Diffusée le 5 mars 1956 à la RDF chaîne nationale.

25. Fonds Pham Van Ky, BnF : $4^{\circ}$-COL-25/34; $4^{\circ}$ Ya 25 Rad. Diffusée le 6 février 1958 à la RDF, club d'essai (SACD). 
26. Fonds Pham Van Ky, BnF : $4^{\circ}-$ COL-25/76 (1-2). Diffusée le 18 mai 1955 à la R.D.F chaîne nationale (SACD).

27. Fonds Pham Van Ky, BnF : $4^{\circ}-\mathrm{COL}-25 / 57$ (1), (2). Diffusée le 18 mars 1950 à la Radio française (SACD).

28. Fonds Pham Van Ky, BnF : $4^{\circ}$-COL-25/47. Présentée au Concours prix Italia, 1959 (Cf. «Fonds Pham Van Ky » à la BnF).

29. Fonds Pham Van Ky, BnF : $4^{\circ}-\mathrm{COL}-25 / 51$. Pièce diffusée le $1^{\mathrm{er}}$ novembre 1955 à la RDF chaîne parisienne (SACD). L'enregistrement est consultable sur http://www.ina.fr/audio/PHD88015101/ la-malediction-des-sept-felicites-audio.html.

30. Ce vocable est indiqué dans la présentation de la pièce La Malédiction de sept félicités sur http://www.ina.fr/audio/PHD88015101/la-malediction-des-sept-felicites-audio.html. Au début de l'émission, Phạm Văn Ký a expliqué la signification de ce mot dans sa conversation avec Pierre Very : Pierre Very : «Cette Quantine-là, pose-t-il la question dans le fait-divers, s'écrit d'ailleurs $q$ $u-a-n$ et non pas $c-a-n$. Est-ce que vous connaîtriez l'origine de ce mot ? » / Phạm Văn Ký : «Oui, c'est-à-dire... il y a des hypothèses très savantes sur la formation de ce mot. Mais, pour ma part, je crois tout simplement qu'il s'agit d'une retranscription, d'une modification, d'une déformation même des deux mots chinois guan yin. » / Pierre Very : «C'est-à-dire ? / Phạm Văn Ký : « Guan yin, guan yin c'est le nom d'une déesse chinoise, la déesse du bonheur justement, selon la tradition populaire. Ce qui recoupe d'ailleurs avec les sept félicités, la Quantine des sept félicités. » 31. "La boîte aux lettres ", in Opéra : l'hebdomadaire du théâtre, du cinéma, des lettres et des arts (de Jacques Chabannes), Paris, 14 mai 1947.

32. Cf. Fonds Pham Van Ky, BnF : $4^{\circ}$-COL-25/112.

33. Cf. Fonds Pham Van Ky, BnF : $4^{\circ}$-COL-25/104.

34. Lode Roelandt et Van Ky Pham, "Phạm Văn Ký écrivain vietnamien », Le Soir, Bruxelles, 5 avril 1954.

35. L'ouvrage source de la pensée chinoise qui, au-delà de sa fonction divinatoire, traite des mouvements de l'univers et de leur rapport avec l'existence humaine.

36. Van Ky Pham, Des Femmes assises çà et là, op. cit., p. 7.

37. Ibid., p. 91.

38. Fonds Pham Van Ky, BnF : $4^{\circ}$-COL-25/91.

39. Hổ Phạm, « Những kỷ niệm về anh tôi ", in Văn Ký Phạm, op. cit., p. 57. Nous traduisons du vietnamien en français.

40. Fonds Pham Van Ky, BnF : $4^{\circ}$-COL-25/15.

41. Fragment de lettre par Phạm Văn Ký, in Fonds Pham Van Ky, BnF : $4^{\circ}-$ COL-25/154.

42. Ibid.

43. Van Ky Pham, Frères de sang, Paris, Éd. du Seuil, 1947, p. 57.

\section{INDEX}

Mots-clés : Phạm Văn Ký, Vietnam 


\section{AUTEUR}

\section{GIANG-HUONG NGUYEN}

Chargée de collections Vietnam, Cambodge, Laos à la Bibliothèque nationale de France 